\title{
Construcción de paz y ciudadanía en la Educación Secundaria y Media en Colombia'
}

\author{
Aura Alicia Cardozo Rusinque ${ }^{2}$ \\ ORCID: 0000-0002-7027-9233 \\ Aldair Ricardo Morales Cuadro ${ }^{2}$ \\ ORCID: 0000-0002-7555-6148 \\ Paola Andrea Martínez Sande ${ }^{2}$ \\ ORCID: 0000-0003-1185-379X
}

\section{Resumen}

El presente artículo tiene el propósito de presentar los resultados de una investigación que buscó establecer una línea de base que permitiera conocer desde la perspectiva de los estudiantes cuáles eran sus apreciaciones sobre el manual de convivencia, el desarrollo e implementación de la cátedra de la paz y la mediación de conflictos dentro de las instituciones educativas con el propósito de desarrollar una construcción de paz estable y duradera, entendida esencialmente como un proceso orientado por el Estado que busque promover buenas prácticas en el aula, formación docente de calidad y estructuras organizativas que favorezcan el aprendizaje de la ciudadanía en el contexto educativo. La investigación está enmarcada bajo el paradigma cualitativo, se realizaron entrevistas grupales semiestructuradas tipo grupo focal con estudiantes de los grados 9, 10 y 11, de diferentes instituciones educativas. Los resultados dan cuenta de la percepción que tienen los estudiantes en las dimensiones evaluadas, siendo relevante: que solo utilizan de manera instrumental el manual de convivencia, y no como fuente de formación; el desconocimiento de los contenidos de la Cátedra de la Paz y de su importancia para contribuir a la construcción de una cultura de paz y el uso por parte de los estudiantes de una autoridad vertical tradicional para resolver los conflictos.

\section{Palabras clave}

Convivencia - Paz - Mediación del conflicto escolar.

1- Investigación realizada sobre actitudes frente a la formación ciudadana, convivencia escolar y construcción de paz en estudiantes de $9^{\circ}$ a $11^{\circ}$ en Barranquilla, Colombia.

2- Universidad de la Costa, Barranquilla, Colombia. Contactos: acardozo@cuc.edu.co; amorales10@cuc.edu.co; pmartine2@cuc.edu.co. 


\section{Peace and citizenship construction in Middle and High Education in Colombia}

\section{Abstract}

This article is intended to present the results of an investigation that pretended to establish a baseline that would allow to know from the perspective of the students which were their appreciations about the coexistence manual, the development and implementation of the chair of peace and conflict mediation within educational institutions with the purpose of developing a stable and lasting peacebuilding, essentially understood as a state-oriented process that seeks to promote good manners in the classroom, quality teacher training and organizational structures that consolidate the learning of the citizenship in the educational context. The research is framed under the qualitative paradigm, semi-structured group interviews focus group type were conducted with students from grades 9, 10 and 11, from different educational institutions. The results account for the perception that students have in the evaluated dimensions, being relevant: that exclusively they use the coexistence manual instrumentally, and not as a source of training; the ignorance of the contents of the Chair of Peace and its importance to contribute to the construction of a culture of peace and the use by students of an Traditional vertical authority to resolve conflicts.

\section{Keywords}

Coexistence - Peace - Mediation of scholar conflict.

\section{Introducción}

El ser humano como ser social convive con otros en diversos ambientes cada vez más complejos, que demandan ambientes equilibrados y sustentables hacia la existencia de un entorno ideal para el fortalecimiento del tejido social y un desarrollo sostenible (UNESCO, 2015). Los contextos históricos, políticos y sociales demarcan de manera significativa la forma como nos relacionamos, como asumimos las normas para lograr convivir en sociedad (BLANCO; VARELA, 2007).

Para consolidar este propósito, la educación debe promover buenas prácticas en el aula, formación docente de calidad y estructuras organizativas que favorezcan el aprendizaje de la ciudadanía (BOLÍVAR, 2007). Partiendo de un conjunto de interrelaciones encaminadas a lograr objetivos a nivel educativo (ROMAGNOLI; MENA; VALDÉS, 2007), asumiendo el conjunto de códigos basados en elementos de carácter ético y moral, con el fin de instaurar una cultura de la convivencia (CAMPS \& GINER, 1998), que involucre aspectos como el respeto por el otro y el ejercicio de la libertad dentro del marco de la justicia (UNESCO, 2008). Sin embargo, 
la convivencia no es un proceso fácil, se ve afectada por la lucha generada frente a recursos limitados, el deseo de dominar a los demás y el egoísmo de los seres humanos (CAMPS; GINER, 1998).

Dentro de los contextos educativos la convivencia se enmarca en la vida que comparten las personas, que demanda entendimiento y comportamientos activos, creadores y empáticos (MARRUGO-PERALTA et al., 2016) dirigidos a generar espacios de aprendizaje mediados por el compartir en donde se adquieren los valores y habilidades sociales, así como las buenas prácticas de convivencia (MEGÍAS, 2011). Así la escuela puede constituirse en un espacio de protección frente a la adversidad del entorno y de la violencia que pueden rodear los escenarios escolares. Sin embargo, la diversidad de factores hostiles presentes en el contexto escolar crea un incremento en la presencia de conflictos entre diferentes actores de la comunidad educativa (BUSTAMANTE, 2014), creando así inquietudes respecto a las causas del repunte de la violencia y de las dificultades en la implementación de las leyes y políticas de convivencia en las instituciones educativas.

Colombia, en la búsqueda de la consolidación del posconflicto, viene desplegando un proceso de tránsito hacia una sociedad normalizada, donde se espera replantear las formas nocivas de relacionamiento, aspiración que nace desde la Constitución Política de 1991, donde se da prioridad a la convivencia y a la paz como fundamentos de la construcción de la nación.

Ante condiciones sociales adversas, donde las personas sufren el impacto directo e indirecto de múltiples formas de violencia, se aprende a configurar las relaciones interpersonales a partir de la imposición del poder y la fuerza, afectando la convivencia y el bienestar (MARTÍNEZ-GONZÁLEZ; AMAR-AMAR, 2017). Esto es particularmente evidente en el contexto colombiano sometido a un conflicto sociopolítico que ha delineado la historia por más de medio siglo, impactando la vida cotidiana (PÉREZ, 2014; PATIÑO, 2014) y permeando las relaciones familiares, escolares y comunitarias.

Ante este desafío, el contexto escolar emerge como escenario donde se ven reflejadas las dinámicas sociales, tanto del conflicto como de los propósitos por construir una cultura de paz, orientadas desde los órganos de gobierno que mediar en la convivencia escolar. Al respecto, el gobierno colombiano establece la Ley 115 de 1994 (COLOMBIA, 1994) o Ley General de Educación y la Ley 1620 del 2013 (COLOMBIA, 2013) mediante la cual se crea el Sistema Nacional de Convivencia Escolar y Formación para el Ejercicio de los Derechos Humanos, la Educación para la Sexualidad y la Prevención y Mitigación de la Violencia Escolar. Con ellas se establecen los mecanismos de formación que se constituyen en un escenario de implementación a través de un manual de convivencia y la organización de los Comités de Convivencia Escolar. Posteriormente con la Ley 1732 de 2014 (COLOMBIA, 2014) establece la Cátedra de la Paz que busca fomentar el desarrollo de competencias relacionadas con el territorio, la cultura, el contexto económico y social, y la memoria histórica, a fin de contribuir a la reconstrucción del tejido social, la prosperidad general y el cumplimiento de los principios consagrados en la Constitución. 
Se entiende que convivir es vivir juntos entre distintos sin los riesgos de la violencia y con la expectativa de aprovechar fértilmente nuestras diferencias (MOCKUS, 2002). Así, el Ministerio de Educación Nacional (COLOMBIA, 2014), define la convivencia escolar como la acción de vivir en compañía de otras personas de manera pacífica y armónica, en busca del desarrollo integral, reconociendo la relevancia de los procesos convivenciales como base para el desarrollo social y el ejercicio de la ciudadanía, y a la escuela como el fundamento para una formación que desarrolle habilidades para saber vivir en paz y de manera constructiva en la sociedad (AL TABLER0, 2004; MARRUGOPERALTA et al., 2016).

\section{Método}

Se realizó una investigación cualitativa, partiendo de categorías de análisis establecidas de forma deductiva a partir de lo establecido sobre la convivencia escolar en los parámetros de ley, con estudiantes de $9^{\circ}, 10^{\circ}$ y $11^{\circ}$ grado, pertenecientes a ocho instituciones educativas del Área Metropolitana de Barranquilla Colombia, que aceptaron participar en el estudio (asentimiento informado) y tuvieron el consentimiento informado de sus padres. La muestra se seleccionó de forma intencional y su aplicación se desarrolló entre los meses de septiembre de 2018 a febrero de 2019.

Como técnica de recolección de información se escogió el grupo focal, técnica de recolección de datos donde mediante una entrevista grupal semiestructurada se analiza el tema propuesto (ESCOBAR; BONILLA, 2017). En este caso, se discutió su percepción sobre la forma como se implementan en sus colegios las competencias ciudadanas, la convivencia escolar y la construcción de paz. Los grupos focales contaron con la participación de entre diez y doce estudiantes.

La información recogida se procesó con el software Atlas ti. Se empleó el análisis categorial de información, el cual recurre a la producción de códigos que permiten ordenar, sintetizar y analizar la información sobre la base de una codificación teórica, realizando la categorización en primera instancia a partir del inventario con el fin de aislar y clasificar los elementos, para luego hacer la clasificación, que permite distribuirlos y organizarlos a partir de la determinación de pautas emergentes con el fin de realizar una aproximación interpretativa y luego la comparación de los resultados a la luz de la teoría (RUIZ-OLABUÉNAGA, 2012).

Para responder a los criterios de fiabilidad y validez, se tuvo en cuenta lo señalado por Porta y Silva (2003), quienes apuntan que la fiabilidad se estima calculando el porcentaje de veces que varios codificadores independientes coinciden al codificar el mismo material. Las categorias reúnen las siguientes características: fueron revisadas con exhaustividad y con homogeneidad dado que sus definiciones se realizaron de acuerdo a un mismo principio de clasificación y responden a pertinencia, objetividad, fidelidad y productividad, proporcionando resultados que enriquecen la investigación. 
En el apartado siguiente se presentan y analizan algunos fragmentos representativos de las respuestas brindados por los participantes en la investigación, transcritos de manera textual.

\section{Resultados}

\section{Categorías emergentes en relación al manual de convivencia}

Los avances en los procesos enmarcados en el diseño de leyes y políticas públicas dirigidas a fortalecer la convivencia escolar se ven representados por el artículo 87 de la Ley 115 de 1994, la cual orienta la creación de un manual de convivencia, que debe ser efectuado en los establecimientos educativos. Presenta los derechos y obligaciones de los estudiantes al momento de la matrícula, el cual debe ser aceptado por los jóvenes y los padres. Sin embargo, su firma se constituye más en un proceso formal, y no en un estudio de su contenido, por lo que finalmente desconocen las normas que orientan sus comportamientos y relaciones e incluso se enfoca más en el derecho que en el deber (MONTAGUT NAVAS; MONTAGUT MANTILLA, 2014). Así lo evidencia un participante cuando se afirma que: "El manual de convivencia es importante porque por medio de este se establecen las reglas del colegio, pero el problema es que la mayoría de los estudiantes no respetan esas normas".

Los participantes declaran que conocen la existencia del Manual de Convivencia e inclusive reconocen que algunos aspectos son importantes para su formación en el campo de la educación, aunque suelen acudir a él de manera instrumental, es decir, lo usan con el fin de hacer valer algunos derechos frente a la autoridad y no como una oportunidad para avalar la convivencia, tal como lo afirma un estudiante: "La mayoría de las cosas que sé del manual de convivencia es lo que me conviene, no lo que debería saber,... como cuando los profesores son injustos".

Esta falta de apropiación se reduce más a una lectura simplificada de que se puede hacer como marco para evitar la sanción, sin empoderar el sentido mismo de la norma y sin comprender su relevancia como un mecanismo para vivir en sociedad, sacrificando el contenido ético, reflexivo y posibilitador de la libertad y la autonomía de actuar mediante una opción por lo bueno en sí mismo (SAVATER, 1991). Muestra de ello se ve plasmado en la siguiente participación

El manual de convivencia nos ayuda un poco para saber, si hago algo malo me puede llevar a una sanción, entonces no lo voy a hacer. Si no lo conozco no sé las consecuencias. En cambio, si yo sé, como dicen por ahí, guerra avisada no mata soldado.

Además, algunos estudiantes manifestaron que se sienten inconformes con las normas, como la expresada por un estudiante quien dice:

Pero en la parte de convivencia hay ciertos aspectos que considero personales. como la forma de presentación personal, no solo el uniforme, sino el cabello, llevar un piercing o lo que sea. Pienso 
que una niña con el cabello rosado, no va a interferir con sus estudios siempre y cuando no le dé una (mala) imagen a la institución.

Para los estudiantes el manual de convivencia está asociado a componentes donde se da prioridad a normas de comportamiento de carácter formal más que con elementos de fondo como el convivir con el otro, la ética y las competencias ciudadanas o la promoción de la ciudadanía (JIMENEZ; LLERAS; NIETO, 2010).

Sin embargo, los estudiantes reconocen que el manual de convivencia promueve valores, como el "liderazgo, responsabilidad y creatividad también" además del esfuerzo de las instituciones para que se cumpla lo estipulado, lo cual, según los estudiantes, es difícil de cumplir por las dificultades para el manejo de los conflictos, el cumplimiento de las normas y el ejercicio vertical del poder en el contexto escolar (BUSTAMANTE, 2014), tal como lo manifiesta una estudiante quien plantea: "Aunque es imposible cumplir todos los valores, porque igualmente estamos en un ambiente escolar con compañeros, profesores y amigos. El colegio se esfuerza por cumplir todos los valores y se le nota”.

Este esfuerzo se evidencia en los primeros cursos en temas asociados a la formación en valores. Sin embargo, se presenta un distanciamiento entre lo que representan los valores y su práctica en la vida ignorando las características de los jóvenes, estos afirman que "lastimosamente, se han ido quedando obsoletos". Así se observa que no logran reconocer las nuevas dinámicas de los adolescentes, quienes se plantean nuevos valores o resignifican los existentes manifestados en procesos de integración e inclusión con grupos poblacionales específicos, un cambio en el manejo de la sexualidad y la manera de relacionarse, incluyendo fenómenos actuales como las redes sociales (CÁRDENAS RUIZ, 2016). Así se puede ver en la siguiente afirmación.

Se trabajan en los cursos de primaria, pero son desarrollados sin establecer un significado para ellos, además que se queda en el conocimiento, más no se llevan a la práctica, ni a la interiorización de los mismos.

Desde la perspectiva de los estudiantes la formación en valores en secundaria deja de ser sistemática, en tanto las instituciones no se preocupan por promoverlos y posibilitar su puesta en práctica.

Con relación a los procesos de participación en la construcción del manual de convivencia algunos estudiantes reconocen que se presentó la participación activa de los estudiantes en la construcción del manual de convivencia, pero consideran que solo se implementó una vez fue reglamentada, ahora refieren que tienen poca incidencia en el manual, tal como lo afirman los siguientes participantes:

Está el que representa los padres de familia, las directivas, la psicóloga del colegio, uno de los profesores, el de puesto más alto supongo, el personero y el representante de once grado, Igualmente 
cada curso tiene su representante que es elegido mediante votación y este ayuda a tomar decisiones, aunque en este colegio tampoco se le da la importancia que se merece para la representación del curso... no se le da mucha importancia ni a la opinión, o a los aportes que pueda dar.

Solo algunas veces tienen la posibilidad de conocerlo y trabajarlo en las aulas de clase, tal como lo afirma un estudiante "Creo que lo hicieron las directivas y algunos padres de la asociación de padres aquí en el colegio, pero estudiantes y eso no estoy segura”. Lo que genera un débil empoderamiento de los contenidos del manual de convivencia, y es asumido como una imposición de las fuentes de autoridad en los contextos escolares, lo que es expresado así por una participante:

Y aparte de eso no piden opiniones al respecto o tal vez si lo hacen en el comité de directivos, pero no es algo en donde todos los estudiantes podamos participar. El personero no nos pregunta cosas como: ¿Están de acuerdo con esto?, así que no siento que haya una participación o voz por parte del estudiante hacia el manual de convivencia.

Frente a esta situación, los estudiantes consideran que el manual de convivencia se trabaja con menos relevancia actualmente, expresando que con el paso del tiempo tienen menos participación generando falta de apropiación de las normas establecidas, distanciándose del propósito de contribuir en la construcción del contexto escolar como escenario propicio para la convivencia (MONTAGUT NAVAS; MONTAGUT MANTILLA, 2014). Percepción que presenta un participante en el siguiente texto:

En épocas pasadas en la institución se daba que colocaban el manual de convivencia en las clases de sociales para que compartiéramos y habláramos sobre las reglas planteadas que estaban ahí y saber qué tipos de reglas son, para que nos sirven, que nos puede llegar a suceder si no las cumplimos, pero en estos momentos ya no se hace.

Situación que demuestran la pérdida del lugar que se ha promovido a las figuras relacionadas al gobierno escolar, quienes han pasado a jugar un papel pasivo en la dinámica propia de la convivencia y la representación estudiantil:

Pienso que, pues no es solamente en este colegio, en el cual no se les da mucha importancia a los personeros ni a los representantes de cada salón, y tampoco pueden cumplir lo que prometen, aunque tengan la mayor intención, van a donde los directivos y le proponen, pero no se interesan en el cumplimiento de las propuestas.

Cabe resaltar que los colegios privados con formación religiosa presentan un mayor trabajo para que los estudiantes conozcan las orientaciones del manual de convivencia, se reconoce que "hay profesores que nos lo repiten y nos lo leen para que nosotros lo tengamos en cuenta”. 
Figura 1- Árbol categorial manual de convivencia

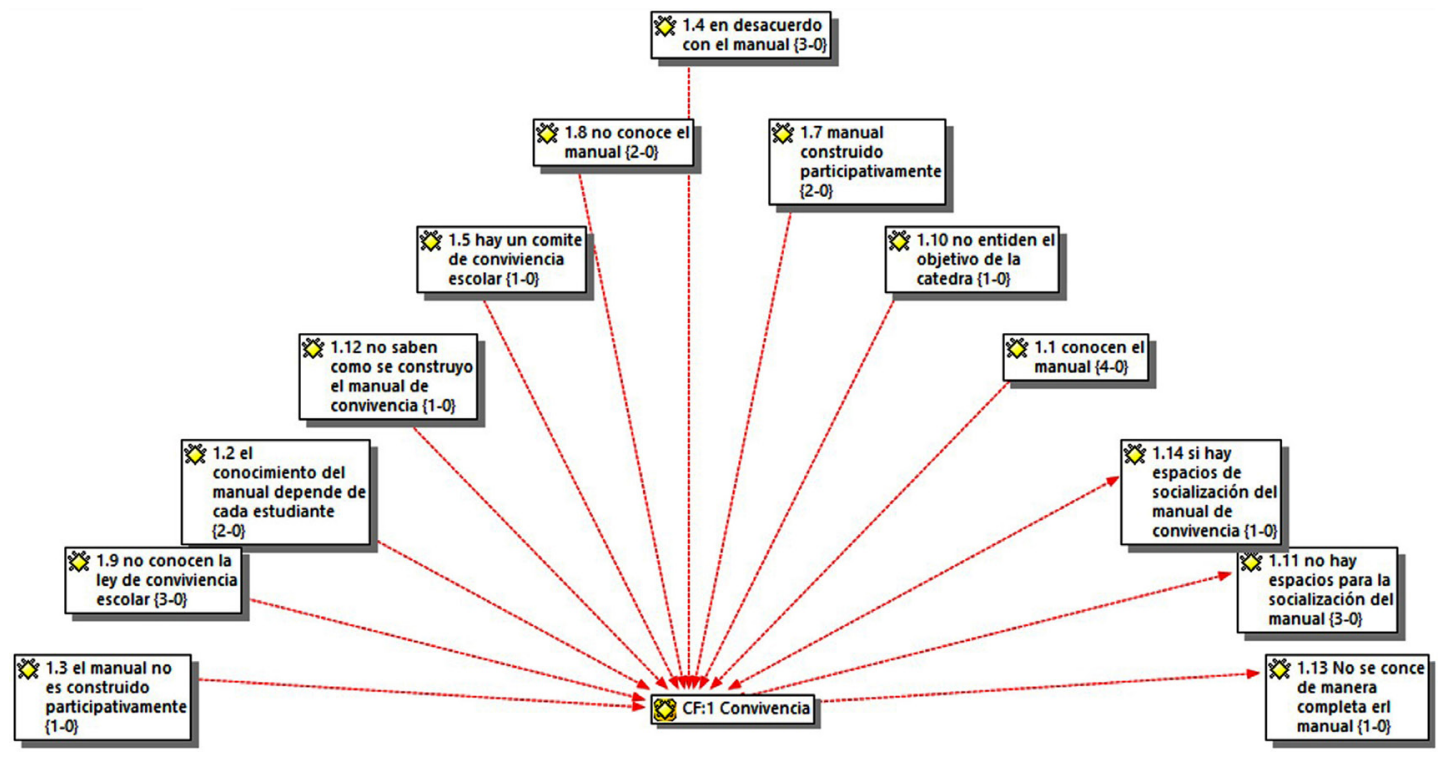

Fuente: Elaboración propia. Aquí se evidencia la falta de conocimiento por parte de los estudiantes de los procesos participativos en la construcción del manual de convivencia, así como del conocimiento de sus contenidos, aunque se reconoce que puede ser socializado, este proceso no logra generar su conocimiento de manera suficiente. De igual manera no se conoce si en los colegios existe un comité de convivencia, apareciendo de manera incipiente esta categoría.

\section{Categorías emergentes en relación al conflicto escolar}

La convivencia escolar se ve afectada por la aparición de los conflictos, los que a su vez suelen ser un reflejo de la dinámica social, ya sea experimentado en el entorno familiar, comunitario o en uno más amplio, como el conflicto armado y el narcotráfico los cuales abonan al fortalecimiento de las pandillas juveniles (MORATTO; CÁRDENAS; BERBESÍ, 2017), aspectos que contribuyen al establecimiento de una cultura violenta, articulada con una debilidad en los procesos de formación en ciudadanía (RESTREP0, 2006).

La solución a los conflictos se presenta a partir de dos formas: mediante el ejercicio del poder en sus diferentes manifestaciones, o mediante el uso de la mediación. Ambas formas funcionan, aunque se favorece la mediación entendida como una serie de competencias encaminadas a la solución pacífica de los conflictos (SILVA; TORREG0, 2016).

Desde esta perspectiva, para atender a la demanda de los fenómenos asociados con la convivencia escolar, requiere conocer cómo se resuelven los conflictos, la implementación adecuada de mecanismos de mediación, reconociendo a su vez las características del contexto que afronta el país (JOJOA PATIÑO, 2016), además de identificar estas diferencias entre conflicto y violencia por parte de los involucrados. Así se encuentra que en las repuestas de los estudiantes participantes hay una clara distinción de estos conceptos, igualmente reconocen que si no se da un manejo adecuado al conflicto, se puede pasar 
a la violencia para solucionarlo (PAREJA FERNÁNDEZ DE LA REGUERA, 2007). Esta diferencia se puede constatar en la siguiente declaración de un estudiante entrevistado

Hasta donde tengo entendido se pasa de conflicto a violencia,... Es la forma mediocre que tiene el conflicto de ganar... Yo pienso que la violencia tiene un grado más alto que los conflictos, es como un roce de ideas que tu no aceptas, pero la violencia es más a los golpes y a la fuerza.

Para los estudiantes los conflictos e incluso la violencia se pueden presentar entre compañeros, pero también entre docentes y directivos con los estudiantes, afectando la forma de relacionarse en el contexto escolar. Tal como lo expresa un participante:

Yo tuve un conflicto con una profesora, ella me pidió explicación de por qué había llegado tarde, y yo al momento de responderle, comenzó a gritarme y a decirme que no le diera explicación, en un tono sarcástico, y yo ya no respondí, me dejo de hablar, se lo tomó como un insulto, porque no le respondí. No sé cómo quería que le respondiera. No tomo la medida solo contra mí, sino con todo el curso, es la hora y no entiendo por qué hizo eso.

Los estudiantes consideran que las discrepancias con los profesores resultan de muy difícil manejo dado que estos asumen su autoridad de manera vertical, donde las expresiones de los estudiantes en el marco del conflicto son descalificadas y vistas como una confrontación a su posición como profesor, lo que imposibilita el dialogo en tanto niega el reconocimiento del estudiante como sujeto en la interacción (SILVA; TORREG0, 2016).

A nivel de los profesores, siempre hay discusiones. Por ejemplo, tú te equivocas en algo, comienza una discusión con la profesora, y para remediar el problema llegas donde la profesora a pedirle disculpas. Entonces la profe te ignora y le haces una pregunta sobre la clase y te sigue ignorando... Si uno se le acerca a pedirle disculpas ella no tiene que tratarnos así, ella es la maestra, ella tiene que enseñarnos, al igual que nosotros la tenemos que respetar, ella también nos tiene que respetar a nosotros.

También es pertinente mostrar que para los estudiantes los conflictos entre pares afectan la forma de relacionarse en el contexto escolar, aunque cabe resaltar que los estudiantes que participaron en el grupo focal se centraron más en las diferencias con sus profesores y directivos. Definen los conflictos entre estudiantes a partir de las diferencias de opiniones.

Por algunos estudiantes que no tienen la misma manera de pensar o el mismo temperamento, siempre tienden a chocar, puede ser en el salón de clases, o con otros compañeros o con profesores que se dirigen al estudiante de una manera que no lo tome bien y ahí empiezan a chocar.

En el siguiente gráfico se pueden apreciar las categorías emergentes relacionadas con el conflicto, donde se pueden apreciar aspectos como la falta de confianza en las directivas por parte de los estudiantes para la resolución de los conflictos, la falta de 
claridad frente a la figura del mediador, lo que se constituye en una dificultad para solucionar los conflictos de manera satisfactoria, además de la presencia de conflicto entre los directivos del colegio y los estudiantes, en razón de la tendencia a resolverlos por parte de los directivos de manera vertical.

Figura 2- Árbol categorial conflicto

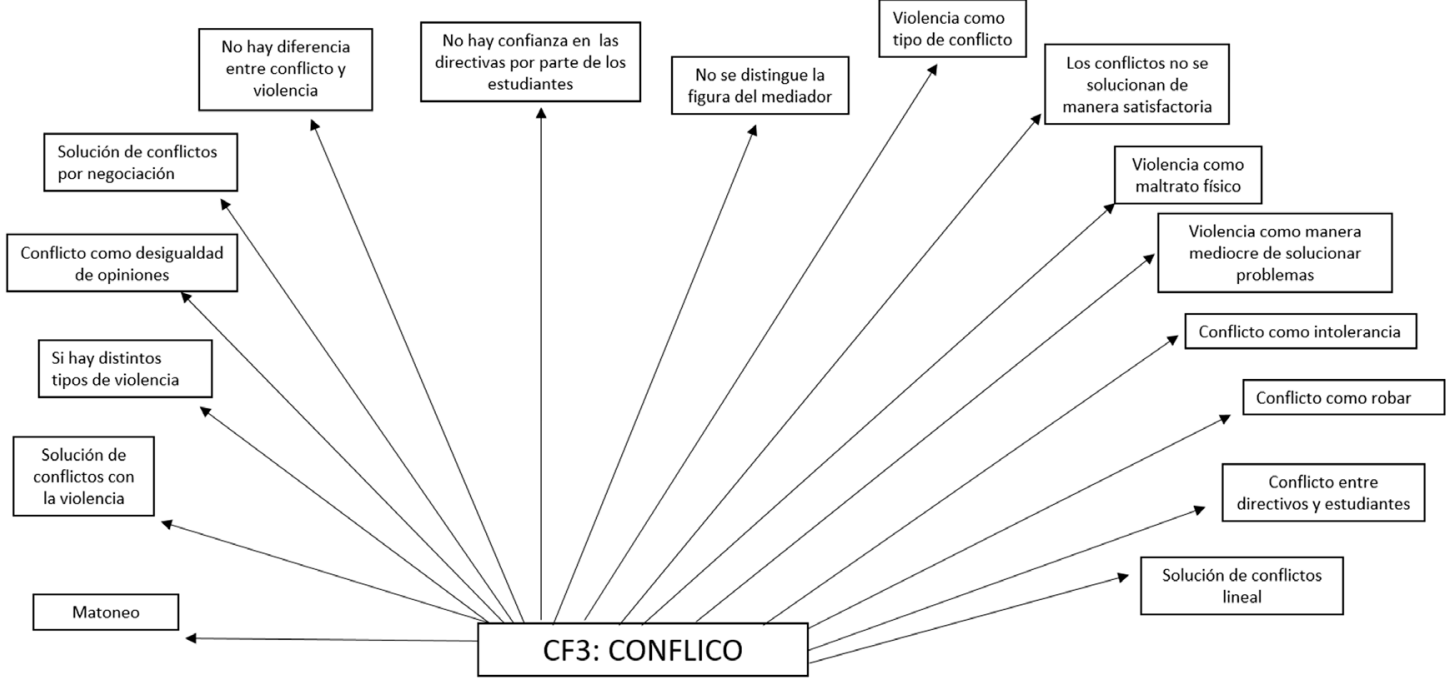

Fuente: Elaboración propia. Se evidencia el reconocimiento de la negociación como una salida a los conflictos en el contexto escolar. Sin embargo, aparece de manera significativa la solución de los conflictos mediante el uso la violencia. Con relación al conocimiento de las diferencias entre conflicto y violencia se evidencia que los estudiantes tienen claro la diferencia.

A partir de la confrontación definida por el poder en la relación docente - estudiante, los procesos de mediación de conflictos en el contexto escolar se ven permeados por esta dinámica, más allá del deber ser presentado por diferentes instancias teóricas y metodológicas para la resolución de conflictos. Aspecto que será desarrollado en el siguiente apartado.

\section{Categorías emergentes en relación a la mediación de conflictos}

Las dificultades para la comprensión del conflicto en el marco de la escuela se ven complejizadas por la difícil combinación de varias formas de violencia que se presentan en la sociedad colombiana, donde el conflicto se ha asociado a la violencia y por tanto, se presenta como un factor necesariamente negativo y no se asocia a una condición propia de las sociedades, ni como una oportunidad para el cambio (SUAREZ-BASTO, 2008). Como consecuencia de esto se dificulta una concepción más positiva del conflicto, lo cual permitiría su control en tanto que se incorpore en la vida cotidiana, formando parte de las relaciones haciendo posible su resolución por la vía no violenta. 
Por tanto, la forma como demos solución a los conflictos en el contexto escolar va a depender de la forma como sean percibidos y la capacidad para generar una acción deliberada para tramitarlos desde el diálogo, la no violencia y el ejercicio de la democracia (HOYOS, 2001). La solución de problemas por la vía no violenta se hace posible cuando se incluyen elementos fundamentales como: entender los conflictos y lo que representa para la comunidad; entender que hay varias rutas que se pueden emprender para su solución; que se deben respetar los derechos humanos, favorecer mejores relaciones basadas en valores y el desarrollo de competencias ciudadanas encaminadas a una forma de vida más productiva en el ámbito de lo público (HOYOS, 2001). El conflicto se aborda no solo para su solución, sino que se constituye en un acto pedagógico de formación para la ciudadanía, la cultura de paz y la mediación de conflictos.

Frente a esta perspectiva, el Estado colombiano ha asumido un proyecto encaminado a formar estudiantes en competencias ciudadanas, que les brinden herramientas para manejar las relaciones con los demás, a superar sin violencia situaciones de conflicto, debatir y encontrar acuerdos de beneficio mutuo mejorando la calidad de vida. Aspectos a desarrollar en el contexto escolar para construir una sociedad pacífica, democrática y respetuosa de las diferencias (AL TABLER0; 2004).

Cuando los conflictos tienden a resolverse a través de la mediación, pueden verse como una posibilidad en la que se entiendan las relaciones humanas desde la comprensión, con una participación activa que intente sostener las relaciones y la satisfacción de las partes a pesar de las diferencias y no desde la búsqueda de juicio o sanción, que afecta la cohesión de los grupos (MARRUG0 et al., 2016). Se promueve el restablecimiento de la comunicación, legitimar los acuerdos y facilitar los momentos de la intervención (MOORE, 1994); propiciar una participación igualitaria, la presencia de un mediador que facilite la búsqueda de soluciones sin imponerlas (HOYOS, 2001). Además de realizar un plan de trabajo, establecer los recursos y legitimar la capacidad de las partes para identificar el problema, buscar alternativas, promover la negociación y formalizar el acuerdo (LEWICKI; SAUNDERS; BARRY, 2011).

Aspectos que no son tenidos en cuenta según los estudiantes participantes en la investigación en tanto consideran que se presentan formas menos estructuradas y más jerárquicas, es decir, se siguen tramitando los conflictos de manera lineal: de profesor a coordinador de disciplina a rector. Esta situación se presenta a pesar de las indicaciones plasmadas en las normas para seguir un procedimiento en la resolución de conflictos escolares, como la propuesta en la Ley 1620, donde se orienta la creación de un Comité Escolar de Convivencia conformado por el rector, quien preside el comité, el personero estudiantil, el docente con función de orientación, el coordinador cuando exista este cargo, el presidente del consejo de padres de familia, el presidente del consejo de estudiantes y un docente que lidere procesos o estrategias de convivencia escolar.

Aquí no hay ningún comité,... si te mandan a coordinación lo hace coordinación, o a bienestar estudiantil, y ahí hay una psicóloga, o una trabajadora social. Pero generalmente es la coordinación, o sea, las decisiones las toma el coordinador y no se tienen en cuenta los demás. 
Los estudiantes ignoran la posibilidad de participar en acuerdos a partir del Comité de Convivencia y de la importancia de buscar alternativas pacíficas a las controversias que se presentan entre ellos o con los docentes y directivos. Lo que dificulta la apertura de los espacios orientados a la solución de conflictos por la falta de apropiación de los elementos conceptuales, técnicos, pedagógicos y administrativos que ofrece la ley 1620 del 2013 (BOCANEGRA; HERRERA, 2017).

Los participantes identifican que hay un designado por parte del colegio para abordar las situaciones conflictivas, que suelen ser el coordinador de disciplina o un psicólogo, particularmente en los colegios privados, los cuales acogen una normatividad interna que limita la presentación de conflictos mediante mecanismos de control y sanción que son relevantes para su permanencia en los establecimientos educativos, por lo que los conflictos son menos evidentes entre compañeros y profesores. Mientras que en los colegios públicos estos mecanismos de control son menos definidos, facilitando la aparición de conflictos que pueden presentarse tanto dentro del centro educativo como en lugares aledaños, tal como lo expresa un participante.

Yo creo que los profesores y las directivas ven que hay un conflicto entre estudiantes, van donde la psicóloga, les hablan de cuál es el problema, y le preguntan qué pasó a cada uno. Hacen el proceso de convivencia, que es firmar un acta, que dice que no va a volver a pasar y se registra en el observador.

Frente a esta situación algunos estudiantes ven como una forma de resolver los conflictos el ejercicio de la violencia, ejerciéndola generalmente fuera de las instalaciones escolares, siendo de mayor presencia en colegios ubicados en barrios vulnerables y en instituciones públicas (HEREDIA; GAVIRIA, 2016).

Entonces eso va como subiendo y subiendo hasta que llegan al extremo de vamos a darnos trompá, en la salida te espero, y se presenta la situación que comienzan a darse puño, entonces por esos conflictos, hay muchas amenazas, porque uno ardido, entonces dicen, ahora que estés por ahí te cojo, hay que ver, pero ellos a veces se ponen a amenazar.

Los estudiantes manifiestan que el contexto escolar se constituye en un lugar de riesgo "Es mejor estar allá afuera que acá adentro, allá afuera es más seguro", a pesar de que los retos para las confrontaciones regularmente se llevan las afueras del centro educativo "Y si comienza una pelea aquí no termina aquí sino allá afuera", esto en razón de que los límites y el ejercicio de la autoridad se ven diluidos por parte de las directivas y maestros, lo cual se acrecienta cuando provienen de hogares que presentan violencia intrafamiliar y de comunidades vulnerables donde las expresiones de violencia suelen ser la forma más valorada para resolver conflictos. (PACHECO-SALAZAR, 2018).

Algunos [conflictos] se resuelven de la mejor manera, otros no. Porque hay estudiantes que no van directamente donde el profesor o la psicóloga, sino que dicen que en la salida arreglamos. Algunos no, algunos hacen lo correcto, van donde el profesor a donde tengan mayor confianza y le dicen profe, en el salón o dentro del colegio me paso esto con tal persona, me amenazó. 
No obstante, hay claridad frente a la importancia de arreglar los conflictos por la vía de la negociación y la mediación especialmente dentro de las instituciones privadas según lo que manifiestan los estudiantes:

Hay diferencias, pero diferencias ya normales entre todos. Ya eso es algo mínimo que se puede solucionar con el diálogo, nunca se ha llevado al extremo.

\section{Categorías emergentes en relación a la cultura de paz}

A lo largo de la historia, mundialmente las sociedades han estado sometidas a guerras y conflictos que han afectado el desarrollo de la humanidad, fracturando el tejido social, y permeado la forma de relacionarnos y de actuar en la sociedad. Frente a esta dinámica de destrucción surgen alternativas que buscan mitigar la difusión de la violencia. El programa Oficina de las Naciones Unidas para el Sostenimiento de la construcción de la paz (PBSO, 2014) define la construcción de paz como las medidas que busquen reducir la probabilidad de caer o recaer en un conflicto, para lo cual se requiere del fortalecimiento de las capacidades en todos los niveles para la gestión de conflictos, que permita afirmar las bases de la paz y el desarrollo sostenible, con el fin de consolidar la paz a partir de las propias necesidades y la consecución de los objetivos.

En concordancia con este propósito se requiere de un conjunto de acciones que permitan a una sociedad prevenir, gestionar y resolver los conflictos, a partir de sus propias capacidades y sin recurrir a la violencia (IECAH, 2010), fortaleciendo su capacidad de crear seguridad y desarrollo sostenible, mediante la mejora estructural de nivel social, económico y político, que encamine sus acciones a prevenir los conflictos, mantener la paz, crear sistemas de alerta temprano, reformar la seguridad, consolidar autoridades legítimas, por tanto al desarrollo humano, teniendo en cuenta las diferencias culturales y del entorno que particularizan los conflictos (ARÉVALO, 2014), pero respondiendo de manera global a la construcción de paz una vez finalizado un conflicto (CÁRDENAS, 2008).

La construcción de paz depende esencialmente de un proceso orientado por el Estado desde el ámbito estructural involucrando a la sociedad en su totalidad, mediante la consolidación de una cultura de paz. Se hace viable a partir de las políticas públicas y de las leyes encaminadas al afianzamiento del posconflicto. Frente a este propósito se ha evidenciado la importancia de la educación para su consolidación, lo que se afianza con la implementación de la Ley 1732 que legisla la Cátedra de la Paz, de obligatorio cumplimiento en todas las instituciones educativas del país, con el fin de generar ambientes pacíficos desde las aulas por lo que debe ser incluida como materia (COLOMBIA, 2015).

El objetivo de la Cátedra de Paz es

[...] fomentar el proceso de apropiación de conocimientos y competencias relacionados con el territorio, la cultura, el contexto económico y social y la memoria histórica, con el propósito de reconstruir el tejido social, promover la prosperidad general y garantizar la efectividad, los principios, derechos y deberes consagrados en la Constitución. (COLOMBIA, 2015, p. 1). 
Los contenidos de la catedra se han especificado como: a) La cultura de paz; b) Educación para la paz; y c) Desarrollo sostenible.

A pesar de la claridad de la Ley y sus decretos, en la implementación de la Cátedra de la Paz se evidencia una débil apropiación por parte de los estudiantes, quienes manifiestan que no hay una claridad sobre lo que se enseña, no cuenta con un espacio específico para su desarrollo. Se articula a asignaturas como Ética y Valores, Ciencias Sociales o en el componente religioso, tal como lo manifiesta un estudiante:

Aunque la materia que está decretada, en la institución no se maneja una buena cátedra de paz, no es una materia sino como los devocionales, educación cristiana, materias como competencias ciudadanas, ética, en los cursos que tenemos ese tema de paz y de reconciliación.

A lo que se agrega que para ellos que son aspectos irrelevantes; repiten temas asociados a la formación en valores o la metodología de los docentes no generan motivación frente a los contenidos de la cátedra y realizando lecturas ocasionales que no resultan significativas, tal como lo manifiesta una participante

Aquí en el colegio, que yo sepa, se manejan mucho más con las lecturas. Este periodo tocó fue la niñez, los derechos de los niños y derechos sociales y civiles eh.... también mucho de valores.

De los colegios de los estudiantes participantes, se ha evidenciado que desde la perspectiva de ellos, no se ha asumido el desarrollo de la Cátedra de la Paz. No obstante, los estudiantes entrevistados reconocen la necesidad de profundizar en temáticas sociales y de conocer la historia reciente para la comprensión de problemáticas sociales, políticas y económicas en Colombia.

Pienso que nos pueden dar esos espacios, nos colocaron en nuestro horario cátedra de paz, y es una materia. Sin embargo, como estudiante, no es como que muchos vayamos a aprovechar ese espacio, para ponerlo en práctica o para conocer.

Además, los estudiantes consideran que los procesos educativos correspondientes a la construcción de ciudadanía y el fortalecimiento de las competencias ciudadanas no llenan sus expectativas, no permitiendo alcanzar los objetivos propuestos en formación en esta temática.

Igualmente, el colegio precisamente es una formación para los estudiantes para salir a una ciudad, a una sociedad competente, y una cátedra de ciudadanía nos vendría muy bien... Siempre es bueno tener espacios en el que estudiante se pueda expresar frente a las diferentes problemáticas, a los diferentes temas de ciudadanía para así seguir creciendo como persona, como sociedad y como estudiantes.

Así mismo, los estudiantes consideran que la principal falencia se encuentra en la metodología de los docentes encargados de la formación en competencias ciudadanas, ciudadanía, ética y valores, llegando a manifestar que no hay diferencia en el contenido curricular de estas asignaturas si se hace una comparación entre lo abordado en la educación básica primaria, educación básica secundaria y educación media como lo manifiesta el siguiente participante: 
Simplemente muchos estudiantes lo veremos como una materia de relleno, que no es importante a pesar de que nos hablan de valores y nuestro comportamiento, de la ciudadanía cuando salgamos a un mundo fuera del colegio. Porque formar un ciudadano, es más bien desde la experiencia, saliendo al mundo y conociéndolo como es. Porque igualmente desde un salón de clases donde te dicen debes actuar de esta manera, no se asimila muy bien. Igualmente, una materia de cátedra de paz, se considera una materia de relleno.

Finalmente, los estudiantes han manifestado un componente que resulta central en sus análisis, que van más allá de la propia dinámica escolar, pero que no le puede ser ajena y es la vida personal y familiar, aspectos que impactan en la cotidianidad y por lo tanto en el contexto escolar, con lo que se complejizan las circunstancias que enmarcan su formación. Parte de esto se ve reflejado en la siguiente intervención que deja en el aire la profunda necesidad de dar respuestas integrales y tanto desde el ámbito estructural como desde el contexto escolar.

Pero no se está tomando en cuenta lo que ocurre en las casas. Por ejemplo, si hay muchos problemas que lo ponen a uno mal, por ejemplo, llegas con una rabia de tu casa y acá te quieres desquitar con todo el mundo, no convives con nadie y el manual de convivencia ahí no vale.

Los participantes presentaron aportes al análisis de lo que es la educación en ciudadanía en sus diferentes categorías, evidenciando que en última instancia recaen en la misma problemática de una instrumentalización de los conceptos que no permiten una trascendencia en la realidad y en sus vidas (VÉLEZ VILLAFAÑE; ORTEGA VALENCIA; MERCHÁN DÍAZ, 2017).

Figura 3- Árbol categorial Cátedra para la Paz

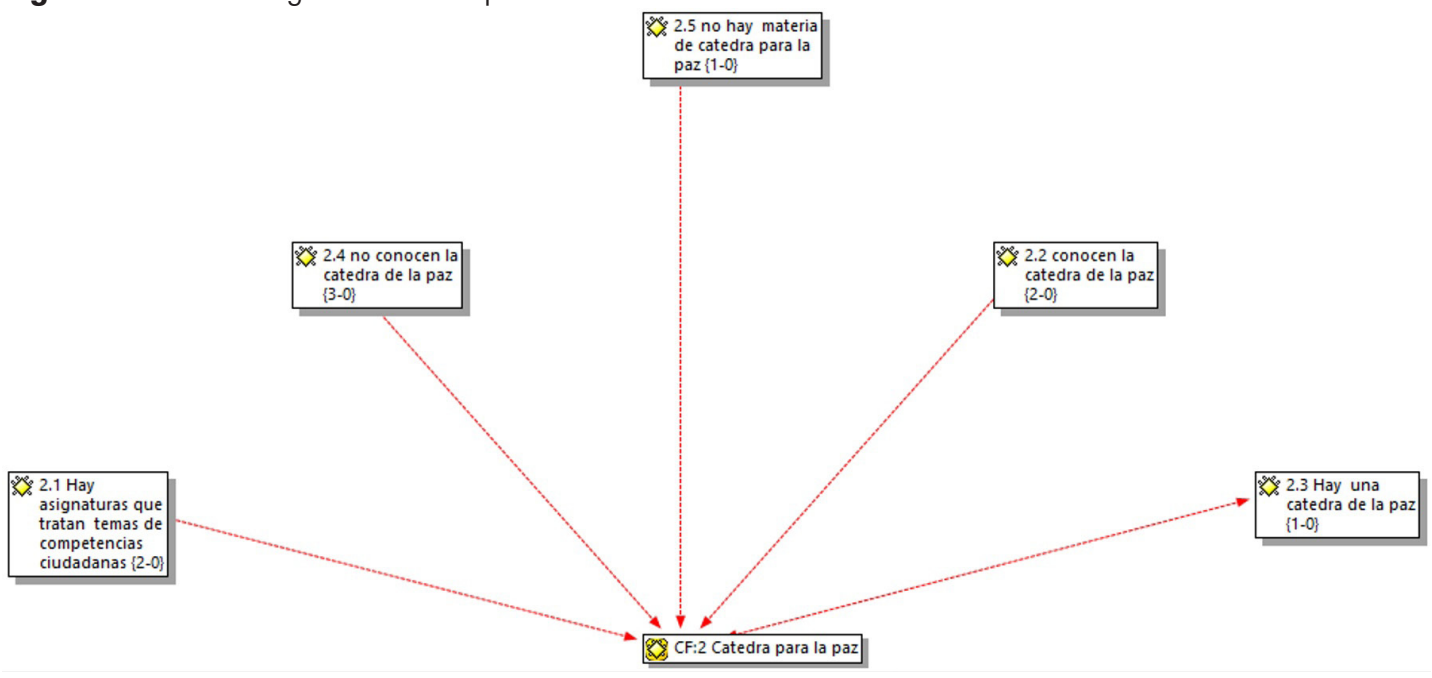

Fuente: Elaboración propia. 
En la figura 3 se presentan las categorías emergentes relacionadas con la Cátedra de la Paz, encontrándose que en algunos casos se desconoce su existencia a pesar de ser orientada mediante una ley que la respalda, por lo tanto, no se ubican de manera específica los contenidos, aunque algunos la identificaron con los contenidos de las clases de Ética y Valores donde desarrollan temas afines.

\section{Conclusiones}

A continuación se presentan algunas conclusiones en torno a las categorías analizadas: convivencia escolar, construcción de paz y mediación de conflictos, donde se evidencia que es un ámbito que demanda estrategias que van más allá de estar reglamentadas para su formación, en tanto para los estudiantes no resulta clara su presencia en el contexto de sus asignaturas, no reviste relevancia para su ejercicio como ciudadanos y no se constituye en una práctica en la cotidianidad de la vida escolar.

Desde lo expresado por los estudiantes se evidencia que el manual de convivencia no representa un insumo importante para orientar de manera integral su comportamiento, resulta ajeno, distante de sus intereses y de las necesidades propias de la adolescencia y no los representa en tanto no participan en su diseño y no son activos en su conocimiento, valoración y apropiación.

Para los estudiantes el manual de Convivencia es un instrumento que solo consultan cuando consideran que pueden usarlo para defenderse de situaciones conflictivas donde se sienten vulnerables, pero no como un ejercicio legitimo de su derecho, más bien como un mecanismo para evitar la sanción, por lo que su uso es básicamente instrumental y no se constituye en sí mismo en un código de comportamiento que posibilite su formación, la legitimación propia como sujetos, ni la legitimación del otro y sus derechos. Se resume las percepciones de los estudiantes con respecto al manual de convivencia en la tabla 1.

Tabla 1- Percepción de los estudiantes con referencia al manual de convivencia

Se desconoce la ley de convivencia escolar

Los estudiantes están en desacuerdo con lo planteado en el manual de convivencia

La mayoría de los estudiantes no conoce el manual de convivencia

Los estudiantes sienten que no tiene espacios donde se socialice el manual de convivencia

Fuente: Elaboración propia.

Los conflictos escolares que se manifestaron con mayor frecuencia son aquellos desarrollados con sus docentes, donde evidencian dificultades para su resolución debido a que las relaciones con ellos aparecen mediadas por una autoridad vertical, negada al diálogo en algunas ocasiones y desconociendo igualmente la legitimidad del estudiante.

Evidencian también que se presentan conflictos con sus compañeros que suelen ser tramitados por la vía de la confrontación directa o la violencia en escenarios externos al contexto escolar, demostrando esta salida como una forma natural de solucionarlos, como si esas diferencias no tuvieran relación con el contexto escolar y por ser presentado como 
personales no tuvieran un espacio para su mediación en el colegio, como se evidencia en la tabla 2.

Se consideran los procesos de mediación de manera muy incipiente, dado que los conductos regulares para su solución siguen siendo verticales, poco participativos y con objetivos claramente sancionatorios, en detrimento de proceso inclusivo y legitimador de los diferentes actores en conflicto y sin mayores aportes para el aprendizaje de un manejo pacífico de los conflictos.

Tabla 2- Percepción de los estudiantes con referencia a la resolución de conflictos

Los estudiantes no logran identificar las diferencias entre violencia y conflicto

Los estudiantes expresan que hay distintos tipos de violencia

Los estudiantes reconocen la negociación como forma de solucionar los conflictos

Los estudiantes sienten que la forma de solucionar los conflictos en sus instituciones educativas es lineal

Los estudiantes no confían en las directivas de la institución

Los estudiantes sienten que están en constante conflicto con sus docentes y directivos.

Fuente: Elaboración propia.

Frente a la Cátedra de la Paz, los estudiantes en general mostraron un desconocimiento de esta materia y de la normatividad que la reglamenta, asociándola con asignaturas como ética y valores, lo cual podría potenciarla, pero no es trabajada con el propósito de promover una cultura de paz que posibilite una formación en ciudadanía activa.

Las categorías analizadas en torno al manual de convivencia, el manejo del conflicto y la mediación y la Cátedra de la Paz evidencian que desde la perspectiva de los estudiantes no abona a su formación integral, se presenta ajena a la dinámica y necesidades de formación en el mundo contemporáneo que responda a los propósitos de consolidar ciudadanos capaces de ser autónomos, libres y éticos.

\section{Referencias}

ARÉVALO, Julián. Construcción de paz y un nuevo modelo de construcción de estado: una lectura de los primeros acuerdos de la Habana. Revista de Economía Institucional, Bogotá, v. 16, n. 30, p. 131-169, 2014. Disponible en: <https://bit.ly/2NZKhiy>. Acceso en: 26 abr. 2018.

AL TABLERO. El periódico de un país que educa y que se educa. Al Tablero, Bogotá, n. 27, febr. 2004. Disponible en: <https://bit.ly/2NSUDk5>. Acceso en: 3 oct. 2018.

BLANCO, Amalio; VARELA, Sergi. Los fundamentos de la intervención psicosocial. In: BLANCO, Amalio; RODRÍGUEZ, Jesús (Coord.). Intervención psicosocial. 1. ed. Madrid: Prentice-Hall, 2007. p. 3-45.

BOCANEGRA, Henry; HERRERA, Carlos. La ley 1620 de 2013 y la política pública educativa de convivencia escolar en Colombia: Entre la formalidad jurídica y la realidad social. Revista Republicana, Bogotá, v. 2017, n. 23, p. 185-214, 2017. Disponible en: <https://bit.ly/2NZKhiy>. Acceso en: 18 my. 2018. DOl: http:// dx.doi.org/10.21017/Rev.Repub.2017.v23.a36 
BOLIVAR, Antonio. Educación para la ciudadanía: algo más que una asignatura. Barcelona: Graó, 2007.

BUSTAMANTE, Nicolás. Los cinco males que atentan contra la Cconvivencia escolar: expertos piden estar alerta frente casos de acoso, discriminación, microtrafico, entre otros. El Tiempo, Bogotá, sep. 18, 2014. Disponible en: <http://www.eltiempo.com>. Acceso en: 11 oct. 2018.

CAMPS, Victoria; GINER, Salvador. Manual de civisme. Barcelona: Ariel, 1998.

CÁRDENAS, Luisa. La construcción de la paz en Colombia: desafíos desde la Escola de Cultura de Pau de Barcelonay la ONU.Bogotá:Universidad MilitarNueva Granada, 2008. Programa de Relaciones Internacionales y Estudios Políticos. Disponible en: <http://repository.unimilitar.edu.co/bitstream/10654/11524/1/ENSAYO DE GRAD0.pdf>. Acceso en: 19 febr. 2018.

CÁRDENAS RUIZ, Juan David. Los medios de comunicación como actores (des) legitimadores: algunas reflexiones acerca del rol de los medios de comunicación sobre la construcción de la opinión pública en torno al proceso de paz de La Habana. Análisis Político, Bogotá, v. 28, n. 8, p. 38-56, 2016. Disponible en: $<$ https://bit.ly/2AeViKg>. Acceso en: 5 febr. 2018.

COLOMBIA. Congreso de la República de Colombia. Ley 115 de 8 de Febrero de 1994. Ley General de Educación, 1994. Diario Oficial, Bogotá, n. 41.214, p. 1-50, 8 febr. 1994. Disponible en: <https://www. mineducacion.gov.co/1621/articles-85906_archivo_pdf>. Acceso en: sept. 6 de 2018.

COLOMBIA. Congreso de la República de Colombia. Ley 1620 de Mazo 15 de 2013, por medio de la cual el gobierno de Colombia crea el sistema Nacional de Convivencia Escolar y la formación para el ejercicio de los derechos humanos, la educación para la sexualidad y a prevención y mitigación de la violencia escolar, del Ministerio de Educación Nacional, 2013. Diario Oficial, Bogotá, n. 48.733, p. 1-19, 15 mar. 2013. Disponible en: <http://www.mineducacion.gov.co/1759/w3-article-322486.html>. Acceso en: 10 oct. 2018.

COLOMBIA. Congreso de la República de Colombia. Ley 1732 de 2014. Por la cual se establece la Cátedra de la Paz en todas las Instituciones educativas del país, 2014. Diario Oficial, Bogotá, n. 49.261, p. 1-3, 1 sept. 2014. Disponible en: <https://bit.ly/1uS17C5>. Acceso en: 14 de nov. 2018.

COLOMBIA. Ministerio de Educación Nacional. Decreto 1038 de 2015. Reglamenta la cátedra de la paz, 2015. Bogotá: Ministerio de Educación Nacional, 2015. Disponible en: <https://bit.ly/2oLn7kH>. Acceso en: sept. 6 de 2018.

COLOMBIA. Ministerio de Educación Nacional. Guías pedagógicas para la convivencia escolar, n. 49. 1. ed. Bogotá: Javier Correa, 2014.

ESCOBAR, Jazmine; BONILLA-JIMÉNEZ, Francy. Grupos focales: una guía conceptual y metodológica. Cuadernos Hispanoamericanos de Psicología, Bogotá, v. 9, n. 1, p. 51-67, 2005. Disponible en: <https:// bit.ly/2LsIOoH>. Acceso en: 3 feb. 2018.

HEREDIA, María Isabel; GAVIRIA, David Andrés. Paz, conflicto y escuela: ¿asuntos del mismo orden? Bogotá: Fundación Convivencia, 2016. Disponible en: <https://fundacionconvivencia.org/apc-aa-files/3f88e0c1cf7 23b0359e2e1758938465b/art-4_2.pdf>. Acceso en: 26 en. 2019. 
HOYOS, Consuelo. La conciliación: un modelo bioético hermenéutico. Medellín: Señal, 2001.

JIMENEZ, Manuela; LLERAS, Juanita; NIETO, Ana. La paz nace en las aulas: evaluación del programa de reducción de la violencia en Colombia. Educación y Educadores, Bogotá, v. 13, n. 3, p. 347-359, 2010. Disponible en: <http://www.redalyc.org/articulo.oa?id=83417001003>. Acceso en: 15 jun. 2018. D0l:10.5294/edu.2010.13.3.2

JOJOA PATIÑO, Johana. Cátedra de la paz: un compromiso con la educación en derechos humanos. Nova et Vetera, Bogotá, v. 25, n. 0, p. 87-98, 2016. Disponible en: <http://esapvirtual.esap.edu.co/ojs/index. php/novaetvetera/article/view/40>. Acceso en: 14 oct. 2017.

IEACAH. Instituto de Estudios sobre Conflictos y Acción Humanitaria. Construcción de la paz. Madri: leacah, 2010. Disponible en: <https://www.iecah.org/index.php/investigacioncp>. Acceso en: 23 nov. 2018.

LEWICKI, Roy; SAUNDERS, David; BARRY, Bruce. Fundamentos de negociación. 5. ed. Madrid: AMGH, 2011.

MARRUGO PERALTA, Guillermo et al. Estrategia de convivencia escolar para la formación de jóvenes mediadores de conflictos. Escenarios, Barranquilla, v. 14, n. 1, p. 72-84, 2016. Disponible en: <https://bit. ly/2La6bSz>. Acceso en: 14 de nov. 2018.

MARTíNEZ-GONZÁLEZ, Marina; AMAR-AMAR, Juan José. ¿Quién es el malo del paseo? Legitimación de la violencia por niños víctimas de migración forzada. Barranquilla: Universidad del Norte, 2017.

MEGÍAS, María Purificación. La convivencia escolar y cómo resolver los conflictos y la violencia en el aula. Granada: Adice, 2011.

MOCKUS, Antanas. Convivencia como armonización de ley, moral y cultura: la educación para aprender a vivir juntos. Revista Perspectivas, Bogotá, v. 32, n. 1, p. 19-37, 2002. Disponible en: <https://bit. ly/2BdMrb9>. Acceso en: 30 oct. 2018.

MONTAGUT NAVAS, Alonso; MONTAGUT MANTILLA, Gersy. Representaciones sociales de lo legal en el ámbito escolar a propósito del manual de convivencia escolar. Justicia Juris, Barranquilla, v. 1, n. 66, 2014. Disponible en: <https://bit.ly/2uSJRCL>. Acceso en: 17 febr. 2018.

MORATTO, Nadia; CÁRDENAS, Natalia; BERBESÍ, Dedsy. Clima escolar y funcionalidad familiar como factores asociadosa la intimidación escolar en Antioquia, Colombia. Pensamiento Psicológico, Medellín, v. 15, n. 1, p. 63-72, 2017.

PACHECO-SALAZAR, Berenice. Violencia escolar: la perspectiva de estudiantes y docentes. Revista Electrónica de Investigación Educativa, Sevilla, v. 20, n. 1, p. 112-121, 2018. Disponible en: <https://doi. org/10.24320/redie.2018.20.1.1523 >. Acceso en: 30 jul. 2018.

PAREJA FERNÁNDEZ DE LA REGUERA, José A. Conflicto, comunicación y liderazgo escolar: los vértices de un triángulo equilátero. Profesorado, Granada, v. 11, n. 3, p. 1-19, 2007. Disponible en: <http://digibug. ugr.es/bitstream/10481/17506/1/rev113COL3.pdf>. Acceso en: 17 dic. 2018. 
PATIÑO, Carlos. Apuntes para una historia de la educación en Colombia. Actualidades Pedagógicas, Cali, v. 64, p. 261-265, jul. 2014.

PÉREZ Tito. Colombia: de la educación en emergencia hacia una educación para el posconflicto y la paz. Riiep, Bogotá, v. 7, n. 2, p. 287-311, 2014. Disponible en: <https://bit.ly/2LtrZ7J>. Acceso en: 25 agto. 2018.

PORTA, Luis; SILVA, Miriam. La investigación cualitativa: el análisis de contenido en la investigación educativa. Red Nacional Argentina de Documentación e Información Educativa, Río Gallegos, p. 1-18, 2003. Disponible en: <https://bit.ly/2AzG9Dh>. Acceso en: 5 mzo. 2018.

PBSO. Oficina de las Naciones Unidas para el sostenimiento de la construcción de la paz. New York: United Nations Peacebuilding Fund / Guidelines on application and use of fundsPBF, 2014. Disponible en: <www. unpbf.org >. Acceso en: 18 dic. 2018.

RESTREPO, Juan. Estándares básicos en competencias ciudadanas: una aproximación al problema de la formación ciudadana en Colombia. Papel Político, Bogotá, v. 11, n. 1, p. 137-175, 2006. Disponible en: <http://www.redalyc.org/pdf/777/77716566006.pdf>. Acceso en: 17 febr. 2018.

ROMAGNOLI, Claudia; MENA, Isadora; VALDÉS, Ana. ¿Qué son las habilidades socio afectivas y éticas? Santiago de Chile: Valoras UC, 2007.

RUIZ-OLABUÉNAGA, José I. Metodología de la investigación cualitativa. 5. ed. Bilbao: Universidad de Deusto, 2012. (Ciencias sociales).

SAVATER, Fernando. Ética para amador. Barcelona: Ariel, 1991.

SILVA, Isabel; TORREG0, Juan Carlos. El equipo de mediación y tratamiento de conflictos como herramienta para resolver conflictos. Impacto sobre los alumnos mediadores en un centro de educación secundaria. In: CASTEJÓN COSTA, Juan-Luis (Coord.). Psicología y educación: presente y futuro. Madrid: ACIPE, 2016. p. 222-279.

SUÁREZ BASTO, Olga Elena. La mediación y la visión positiva del conflicto en el aula: marco para una pedagogía de la convivencia. Diversitas, Bogotá, v. 4, n. 1, p. 187-199, 2008. Disponible en: <https://bit. ly/2MQ5370>. Acceso en: 17 febr. 2018.

TUAY-SIGUA, José. Convivencia escolar como espacio de construcción de ciudadanía en zonas de conflicto y post conflicto. Bogotá: Universidad de Santo Tomás, 2016. Disponible en: <https://bit.ly/2MQ17ng>. Acceso en: 15 en. 2018.

UNESCO. Convivencia democrática, inclusión y cultura de paz: lecciones desde la práctica educativa innovadora en América Latina. 1. ed. Santiago de Chile: Pehuén, 2008.

UNESCO. Rethinking education: towards a global common good? 1. ed. Paris: Uniesco, 2015. 
VÉLEZ VILLAFAÑE, Gabriel; ORTEGA VALENCIA, Piedad; MERCHÁN DÍAZ, Jeritza. La escuela en ecologías violentas: entre las políticas y la pedagogía de la memoria. Revista Latinoamericana de Educación, Bogotá, v. 8 n. 1, p. 187-201, 2017. https://doi.org/10.18175/VyS8.1.2017.11.

Recibido en: 29.10 .2018

Aprobado en: 19.03 .2019

Aura Alicia Cardozo Rusinque es doctora en Psicología. Magíster en Estudios Políticos. Especialista en Estudios Pedagógicos y psicóloga.

Aldair Ricardo Morales Cuadro es maestrante en Psicología con énfasis en Desarrollo Humano y psicólogo.

Paola Andrea Martínez Sande es magíster en Psicología con profundización clínica, psicóloga y filósofa. 